\title{
Liquid-Liquid Phase Separation in an Elastic Network
}

\author{
Robert W. Style, ${ }^{1, *}$ Tianqi Sai, ${ }^{1}$ Nicoló Fanelli, ${ }^{1}$ Mahdiye Ijavi, ${ }^{1}$ Katrina Smith-Mannschott, ${ }^{1}$ \\ Qin Xu, ${ }^{1}$ Lawrence A. Wilen, ${ }^{2}$ and Eric R. Dufresne ${ }^{1}$ \\ ${ }^{1}$ Department of Materials, ETH Zürich, 8093 Zürich, Switzerland \\ ${ }^{2}$ Center for Engineering Innovation and Design, School of Engineering and Applied Sciences, \\ Yale University, New Haven, Connecticut 06520, USA
}

(Received 5 October 2017; revised manuscript received 17 December 2017; published 16 February 2018)

\begin{abstract}
Living and engineered systems rely on the stable coexistence of two interspersed liquid phases. Yet, surface tension drives their complete separation. Here, we show that stable droplets of uniform and tunable size can be produced through arrested phase separation in an elastic matrix. Starting with a cross-linked, elastic polymer network swollen by a solvent mixture, we change the temperature or composition to drive demixing. Droplets nucleate and grow to a stable size that is tunable by the network cross-linking density, the cooling rate, and the composition of the solvent mixture. We discuss thermodynamic and mechanical constraints on the process. In particular, we show that the threshold for macroscopic phase separation is altered by the elasticity of the polymer network, and we highlight the role of correlations between nuclei positions in determining the droplet size and polydispersity. This phenomenon has potential applications ranging from colloid synthesis and structural color to phase separation in biological cells.
\end{abstract}

DOI: 10.1103/PhysRevX.8.011028

Subject Areas: Materials Science, Soft Matter

\section{INTRODUCTION}

Nucleation and growth of liquid droplets is a ubiquitous process. In the sky above us, it underlies the formation of clouds. Inside our own cells, the condensation of proteinrich droplets helps to regulate the translation of RNA, among other cellular phenomena [1,2]. The essential thermodynamics of condensation was worked out in the 19th century by Gibbs [3]. The kinetics of nucleation is more complex and depends sensitively on the presence of impurities [4-7].

A growing appreciation for the biological implications of the phase separation of proteins within living cells [1,8-13] raises a host of questions about the underlying physics [14]. Examples include the role of active processes in determining the properties of phase-separated droplets [15] and the combination of phase separation with kinetic arrest $[10,16]$. Of particular interest here is the interplay of the structure of the cytoplasm and phase separation. The physics of droplet growth has been extensively studied when the surrounding matrix is a simple fluid such as a vapor or Newtonian liquid. However, a living cell has a complex

\footnotetext{
*robert.style@mat.ethz.ch
}

Published by the American Physical Society under the terms of the Creative Commons Attribution 4.0 International license. Further distribution of this work must maintain attribution to the author(s) and the published article's title, journal citation, and DOI. rheology, including significant elasticity from the cytoskeleton $[17,18]$.

Here, we investigate nucleation and growth of liquid droplets inside of a cross-linked polymer network. We show that the resulting droplets are stable and highly uniform, with a size that can be tuned by the cross-linking density, quench rate, and loading of the minority fluid. Condensation in a compliant elastic network is a generic physical process for making monodisperse droplets, and it works for a wide variety of chemistries. We demonstrate its efficacy for both temperature- and composition-driven condensation inside of covalently or physically cross-linked polymer networks swollen with silicone or aqueous solvents. This process may provide a flexible route to the bulk synthesis of monodisperse, polymeric microparticles and nanoparticles and enable the self-assembly of flexible, structurally colored materials $[19,20]$. The interaction of condensation and network elasticity may play a role in the cellular physiology of phase-separated proteins, and the physical parameters identified here could possibly be exploited by living cells to regulate phase separation $[16,21]$.

\section{LIQUID-LIQUID PHASE SEPARATION IN AN ELASTIC NETWORK}

The stability of a fluid mixture depends on the temperature and the concentrations of its components (e.g., Refs. $[6,8,22,23])$. A schematic phase diagram for a typical mixture of two liquids, $A$ and $B$, is shown in Fig. 1(a). Above a critical temperature, any mixture of the two fluids 

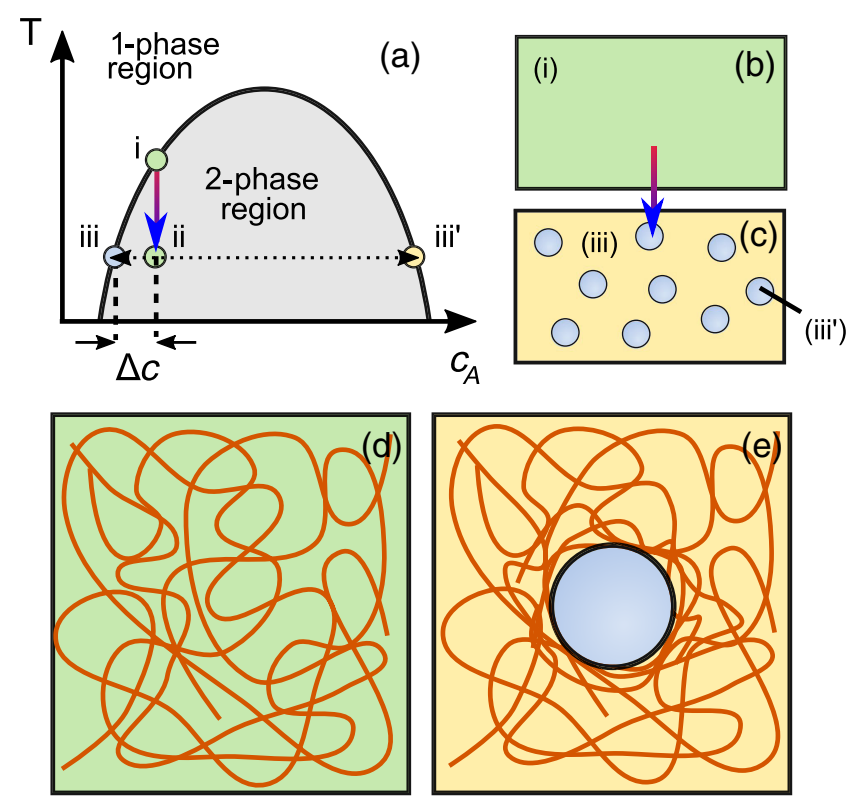

FIG. 1. Phase separation of a liquid mixture in a polymer network. (a)-(c) Schematic diagram of simple liquid-liquid phase separation in a system with an upper-critical solution temperature. Point (i) on the phase diagram indicates a marginally stable mixture where the majority component $B$ is saturated with a dilute component $A$. Upon cooling to (ii), the system spontaneously separates into two phases, a continuous phase (iii) with a low concentration of $A$ and a droplet phase rich in $A$ (iii'). (d,e) Schematics of (d) a polymer network swollen by a mixture of $A$ and $B$ [at point (i) in the phase diagram], and (e) the same system after quenching to (ii) and phase separating. Note that the droplet must deform the network in order to grow beyond the mesh size.

is stable. Below the critical temperature, some mixturesindicated by the gray region in Fig. 1(a)—are unstable. Now consider a marginally stable fluid mixture at the boundary between the stable and unstable regions, as indicated by point (i). When the mixture is cooled rapidly to point (ii) in the unstable region, i.e., "quenched," it spontaneously separates into two stable compositions [Figs. 1(b) and 1(c)]. When the quench is not too deep, separation happens through the nucleation of discrete droplets rich in $A$ (iii'), which grow until the concentration of $A$ reaches its saturation value in the continuous phase (iii). Since the interface between the droplet and the continuous phase costs energy, the droplets tend to coarsen over time. Just like forced mixtures of oil and water, droplets continue to grow until there is a single blob of each phase. This can occur by direct coalescence or through diffusion of the minority component through the continuous phase (i.e., Ostwald ripening).

We investigate how this classic process is modified by the presence of a cross-linked polymer network. Consider a polymer network that is swollen by a mixture of two solvents, $A$ and $B$, as shown in Fig. 1(d). Imagine that this solvent mixture initially lies at point (i) on the phase diagram and is quenched to the unstable point (ii). As before, the fluid mixture needs to separate. For simplicity, assume that the polymer network is excluded by the $A$-rich droplet phase. Provided that the network is not too dense, we might expect that initial nucleation and growth will not be affected by the network. However, once the droplet is comparable to or bigger than the network mesh size, it cannot grow without deforming the network [Fig. 1(e)]. How does this impact phase separation? At a minimum, we expect the network to prevent motion of the droplets, blocking direct coalescence as a route to coarsening. More intriguingly, the thermodynamic forces that drive condensation could be balanced by elastic forces in the polymer network, arresting growth.

When a mixture is quenched into the two-phase regime, it is imbued with excess free energy that can deform a polymer network. Consider an unconstrained liquid in equilibrium with a dilute solute. Here, the solute concentration $c$ will take its saturation value $c_{\text {sat }}(T)$. If $c$ is increased beyond $c_{\mathrm{sat}}$, the solute will condense into the liquid phase until equilibrium is achieved. From ideal solution theory, this is driven by an excess free energy per solute molecule given by $\Delta g(T, c)=k_{B} T \ln \left(c / c_{\text {sat }}\right)$, where $k_{B}$ is Boltzmann's constant (e.g., Ref. [24]). However, if the liquid phase is constrained by the elastic network of a gel, it can have an increased internal pressure $P$. Now, the pressure and volume in the droplet will increase as the solute condenses until the work done by the condensing solute in order to grow the droplet by a solute molecule, $P \Delta V=P M / \rho_{l} N_{A}$ (e.g., Ref. [25]), balances $\Delta g$ (here, $\rho_{l}$ is the density of the liquid, $M$ is the molar mass of the solute, and $N_{A}$ is Avogadro's number). Thus, in equilibrium, we expect

$$
P=\frac{\rho_{\ell} R T}{M} \ln \left(\frac{c}{c_{\mathrm{sat}}}\right)
$$

where $R=k_{B} N_{A}$ is the gas constant (cf. Refs. [26,27]). If the restraining force of the polymer network exceeds this pressure, it cannot grow. This is perfectly analogous to the stalling of a processive molecular motor by a sufficiently large opposing force [28]. Note that these arguments do not rely on phase separation being driven by changes in temperature. Thus, Eq. (1) can also be used to estimate the driving pressure when phase separation is triggered by other processes, such as changes in solvent or solute composition.

Droplets grow freely when they are smaller than the network mesh size, but to grow beyond the mesh size, they have to deform the network. Applying the classic theory of elastic cavitation [29-34] to droplet growth, we expect two regimes delimited by a critical pressure $P_{\text {crit }}=5 E / 6$, where $E$ is Young's modulus of the gel. When $P<P_{\text {crit }}$, a growing droplet should make only modest deformations to the network and will not grow much beyond the network mesh size. However, when $P>P_{\text {crit }}$, droplet growth cannot be stopped by a linear-elastic or neo-Hookean material (e.g., Refs. [31,34,35]) and is only limited by the 
availability of solute. Note, though, that once a droplet is much larger than the mesh size, it will be well beyond the range where we can apply the concepts of linear elasticity. Equivalently, since elastic forces make nucleation and growth energetically unfavorable at small supersaturations, the apparent phase boundary for macroscopic phase separation is shifted:

$$
c_{\text {sat }}^{\mathrm{app}}=c_{\mathrm{sat}}(T) e^{\left[(5 E M) /\left(6 \rho_{l} R T\right)\right]} .
$$

Simple scaling arguments suggest that the condition for solute-limited growth can readily be satisfied in most solvent-swollen polymer networks. Ideal rubber elasticity theory [30] relates Young's modulus to the structure of the polymer network, $E \approx n_{c} k T$. Here, $n_{c}$ is the number density of cross-links in the network. Combining this result with Eq. (1), we find $P / E \approx n_{l} / n_{c}$, where $n_{l}$ is the number density of molecules in the droplet phase [assuming that $\left.\ln \left(c / c_{\text {sat }}\right) \sim O(1)\right]$. Thus, we find that droplets can strongly deform a polymer network whenever $n_{l} \gtrsim n_{c}$ or, equivalently, when the size of a molecule in the droplet is smaller than the mesh size of the polymer network. Polymer networks swollen with a reasonably supersaturated mixture readily meet this condition.

\section{THERMALLY INDUCED PHASE SEPARATION IN SILICONE GELS}

To demonstrate this process, we grow droplets of fluorinated oil in a silicone gel. The silicone gel consists of a cross-linked silicone polymer network swollen in silicone oil. By varying the cross-linking density, we can tune Young's modulus $E$ from about $5 \mathrm{kPa}$ to $1 \mathrm{MPa}$ (e.g., Ref. [36]). We saturate the gels with fluorinated oil at an elevated temperature $T_{e}$. The saturation concentration of the oil in the gel has no significant dependence on the crosslinking density, but it increases with temperature in the range $23^{\circ} \mathrm{C}-55^{\circ} \mathrm{C}$, as $c_{\text {sat }}[\mathrm{wt} \%]=0.093 T_{e}\left[^{\circ} \mathrm{C}\right]+3.2$ (see Ref. [37]). To initiate droplet formation, we cool samples to $23^{\circ} \mathrm{C}$ at a controlled rate.

We estimate the pressure available to deform the elastic network using Eq. (1). For fluorinated oil $\left(\rho_{l} / M=\right.$ $4494 \mathrm{~mol} / \mathrm{m}^{3}$ ), the pressure prefactor $\rho_{l} R T / M=11 \mathrm{MPa}$. In these experiments, we can readily reach $c / c_{\text {sat }}$ up to 2 , corresponding to a driving pressure of $7.7 \mathrm{MPa}$. This exceeds the anticipated threshold for droplet growth, even for the stiffest gels we consider here.

\section{A. Tuning droplet size}

Over a wide range of experimental conditions, we observed uniform micron-scale spherical droplets
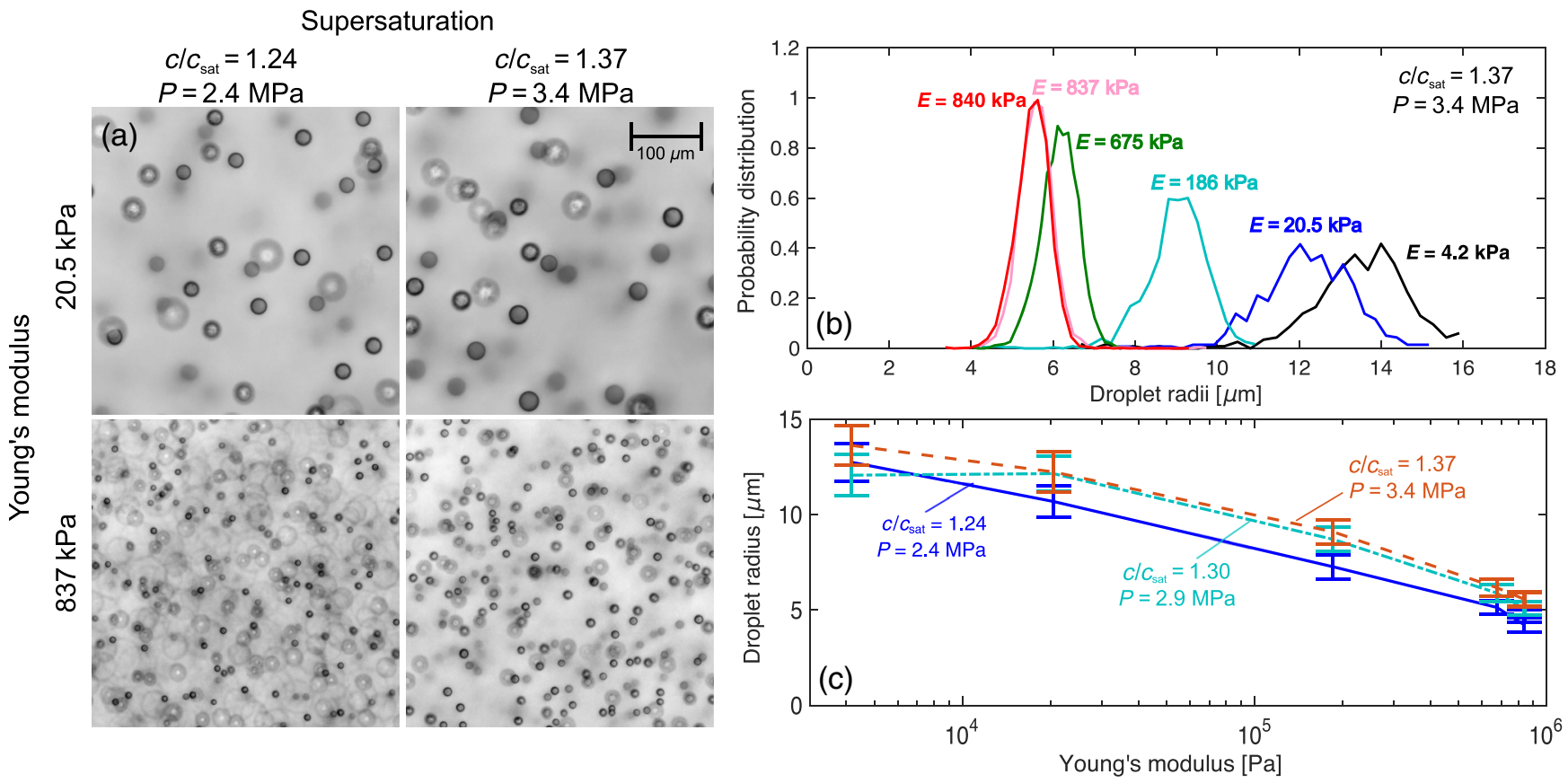

FIG. 2. Droplets formed by phase separation of fluorinated oil in silicone gels. (a) Examples of droplets formed in a soft (top) and stiff (bottom) gels. Examples in the left column have a lower loading than the right column, as they are saturated at $37 / 44^{\circ} \mathrm{C}$ respectively, before being cooled at a rate of $1.5^{\circ} \mathrm{C} / \mathrm{min}$ to $23^{\circ} \mathrm{C}$. Note that some of the droplets appear to have other droplets embedded within them, or some sort of substructure. In fact, this corresponds to the imaging of other droplets that lie behind the current plane of focus. (b) Examples of typical droplet distributions in different stiffness gels, each saturated at $44^{\circ} \mathrm{C}$. (c) The dependence of droplet size on stiffness and saturation temperature. Data points are centered on the average value, and the error bars indicate the standard deviation. In each panel, $P$ is calculated using Eq. (1). 
distributed homogeneously throughout the sample. Typical microstructures of the resulting droplet dispersions are shown in Fig. 2(a), and bright-field optical $z$ stacks are found in Videos 1-4 in Ref. [37]. The droplets are fixed in position and show no visible changes in their radii over time scales of hours. At longer time scales, the droplets shrink as the oil evaporates. Perhaps obscured by the slow evaporation of the oil, we see no evidence for Ostwald ripening or other slow processes. In contrast, droplets of fluorinated oil nucleating and growing in liquid silicone coarsen to the millimeter scale as their density causes them to settle out upon cooling. As described below, the size of the droplets depends on the cross-linking density, the level of saturation, and the quench rate.

The droplet size is primarily controlled by the crosslinking density of the gel. Analysis of the bright-field $z$ stacks allows us to size each droplet and construct size distributions, as shown in Fig. 2(b). In these data, samples are saturated at $T=44{ }^{\circ} \mathrm{C}$ and quenched to $23^{\circ} \mathrm{C}$ at $1.5^{\circ} \mathrm{C} / \mathrm{min}$. This corresponds to a driving pressure of 3.4 MPa. We varied the cross-linking density to achieve Young's moduli from 4.2 to $840 \mathrm{kPa}$. In all cases, the droplets have single-peaked size distributions that are very well approximated by normal distributions with mean $\mu$ and standard deviation $\sigma$. The mean droplet radius varies from about $5 \mu \mathrm{m}$ to $14 \mu \mathrm{m}$. The decrease in mean droplet size with gel stiffness is shown in Fig. 2(c). The dependence on Young's modulus is modest, such that a 200-fold increase in gel stiffness only decreases the droplet size by a factor of 2 .

Droplet size is also impacted by the level of saturation. By varying the incubation temperature, we varied the loading of the oil from $c / c_{\text {sat }}=1.24$ to $1.37 \mathrm{wt} \%$. This $10 \%$ increase in loading (corresponding to a $50 \%$ increase

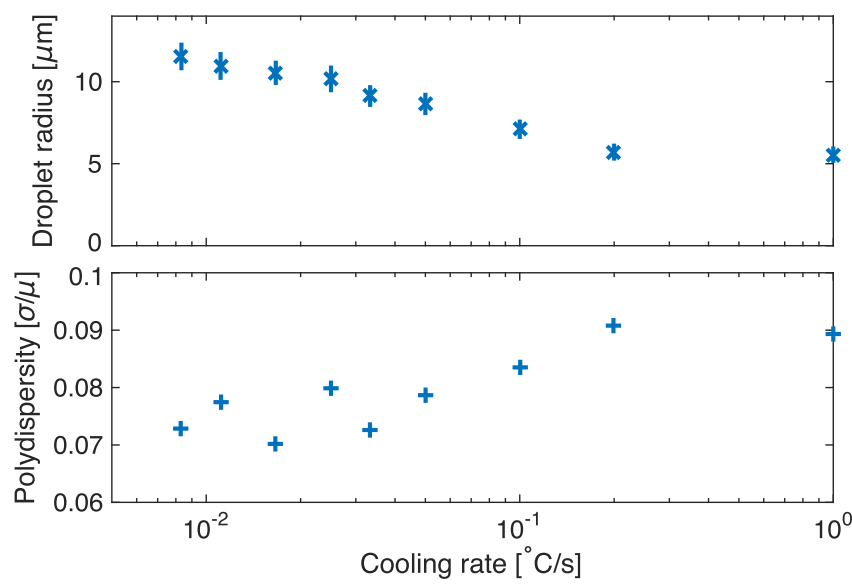

FIG. 3. Rate dependence of droplet size and polydispersity. (Top panel) The mean droplet radius reduces roughly logarithmically with the cooling rate. Here, the error bars are the standard deviation $\sigma$ of the droplet size distribution. (Bottom panel) The polydispersity $(\sigma / \mu)$ is effectively independent of the cooling rate. Note that $E=186 \mathrm{kPa}$, and the sample is saturated at $40^{\circ} \mathrm{C}$. Thus, $c / c_{\text {sat }}=1.37$. in the supersaturation, $c-c_{\text {sat }}$ ) leads to a $25 \%$ increase in droplet radius, as shown in Fig. 2(c).

We can also control droplet size by controlling the cooling rate: Droplets decrease in size as the cooling rate increases, as shown in Fig. 3(a). Again, we find a modest, roughly logarithmic, dependence of droplet radius on cooling rate. Droplets are slightly more uniform when cooled at a slower rate. We quantify the uniformity with the polydispersity index $(\sigma / \mu)$, shown in Fig. 3(b). Over the range of two decades in cooling rate, the polydispersity ranged from $7 \%$ to $9 \%$. Thus, we can control the size with surprisingly little penalty in droplet uniformity as the cooling rate increases.

\section{B. Optical properties of composites with uniform droplets}

The uniformity of the droplet size is reflected in the macroscopic optical properties of the gel. Figure 4(a) shows the scattering pattern in transmission for a thin section of silicone gel containing fluorinated-oil droplets, illuminated with a HeNe laser. There is a clear ring at $6.5^{\circ}$, consistent with the expected Mie scatting pattern for monodisperse spheres [38]. In fact, numerical solutions of the Mie scattering problem predict such a ring for droplets of radius $3 \mu \mathrm{m}$ [39], consistent with our droplet observations (note this sample was cooled 45 times faster than the samples in Fig. 2). There is no significant contribution to the observed scattering pattern from correlations in the droplet locations (i.e., structure factor). Since the silicone gels are highly elastic, we can deform the droplets by stretching the gel [36]. As shown in Video 5 (Ref. [37]), this yields a corresponding stretching of the scattering pattern in the direction perpendicular to the applied stretch-indicating the coupling of the macroscopic deformation to the shape

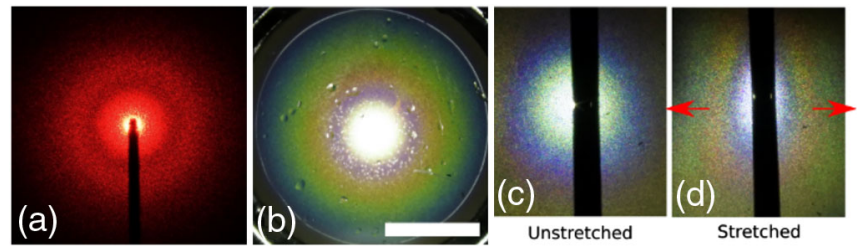

FIG. 4. Optical properties of self-assembled composites of uniform fluorinated-oil droplets in a silicone-gel matrix. (a) The light-scattering pattern of a helium-neon laser by a sample $(E=837 \mathrm{kPa}$ silicone gel saturated with fluorinated oil at $40^{\circ} \mathrm{C}$ and cooled at $30^{\circ} \mathrm{C} / \mathrm{min}$ ) has a clear ring, indicating droplet uniformity. The center of the dark ring is at a scattering angle of $6.5^{\circ}$. The vertical, dark line is a beam stop. (b) Different wavelengths of light are scattered to different angles, leading to colorful rings when a sample (Sylgard 184, $E=40 \mathrm{kPa}$, cooled from $42{ }^{\circ} \mathrm{C}$ at $6{ }^{\circ} \mathrm{C} / \mathrm{min}$ ) is illuminated from behind with a whitelight LED. The scale bar is $10 \mathrm{~mm}$ long. (c,d) The color pattern is deformed upon stretching (Sylgard 184, E=412 kPa, cooled from $42{ }^{\circ} \mathrm{C}$ at $6{ }^{\circ} \mathrm{C} / \mathrm{min}$ ). Here, the direct light from the whitelight LED source is blocked by a 2 -mm-thick rod to better visualize the colors. See Video 2 in Ref. [37]. 
of the microscopic droplets. Different wavelengths are scattered to rings of different radii by the samples, so we observe a pattern of color when a point source of white light is viewed through the sample [Fig. 4(b)]. This pattern changes as the sample is stretched and the microstructure is deformed, as shown in Figs. 4(c) and 4(d) and Video 6 (Ref. [37]). Note that this approach to generating straindependent color is similar to previous work where colloidal particles were embedded in a soft silicone elastomer [40].

\section{Correlated nucleation and growth}

As expected for gentle quenches far from the critical point, droplets form by subsequent nucleation and growth (e.g., Refs. [41,42]). Video 7 in Ref. [37] shows a typical example. Here, $E=186 \mathrm{kPa}$, and the sample is cooled from $42{ }^{\circ} \mathrm{C}$ to $22{ }^{\circ} \mathrm{C}$ at $2{ }^{\circ} \mathrm{C} / \mathrm{min}$. New droplet nuclei appear over a period of about $150 \mathrm{~s}$ and grow over a longer interval, of about $800 \mathrm{~s}$. This suggests that nucleation and growth can be thought of as two separate stages of droplet formation [7]. Here, we show that while these two processes are separated in time, they are strongly coupled in a manner that impacts the droplet size distributions.

Since droplets are trapped in an elastic matrix, nucleation positions are given by the final positions of the droplets. We identified the position of each nucleus using the same bright-field $z$ stacks underlying Fig. 2. First, we determined the number density of droplets, $n_{d}$, as a function of crosslinking density and supersaturation. As shown in Fig. 5(a), $n_{d}$ increases linearly with Young's modulus, but it is independent of the supersaturation. By a simple application of mass conservation, this variation in nucleation number density fully accounts for the dependence of the mean droplet size on the cross-linking density and extent of supersaturation (see Ref. [37]).

In classic theory, nucleation events are typically assumed to be independent. However, we find that the locations of nucleation sites are significantly correlated and central to the form of the final droplet distribution. We quantified spatial correlations in the nucleation positions using the radial distribution function $g(r)$, as shown in Fig. 5(b) for typical experimental data. The pair-correlation function compares the probability of observing two objects at a given separation, relative to a case where all objects are placed perfectly randomly. We find that the number density of closely neighboring droplets is strongly reduced over a distance comparable to the mean interdroplet spacing, about $40 \mu \mathrm{m}$ in the example of Fig. 5. This suggests that there is some mechanism that prevents droplets from nucleating near each other. Two possible reasons for this are the depletion of the dissolved droplet phase and the compression of the polymer network around nuclei, which both hinder further nucleation.

Intriguingly, variations in the local spacing of nuclei have a significant impact on the size distribution. We characterized the structure about each nucleus using the
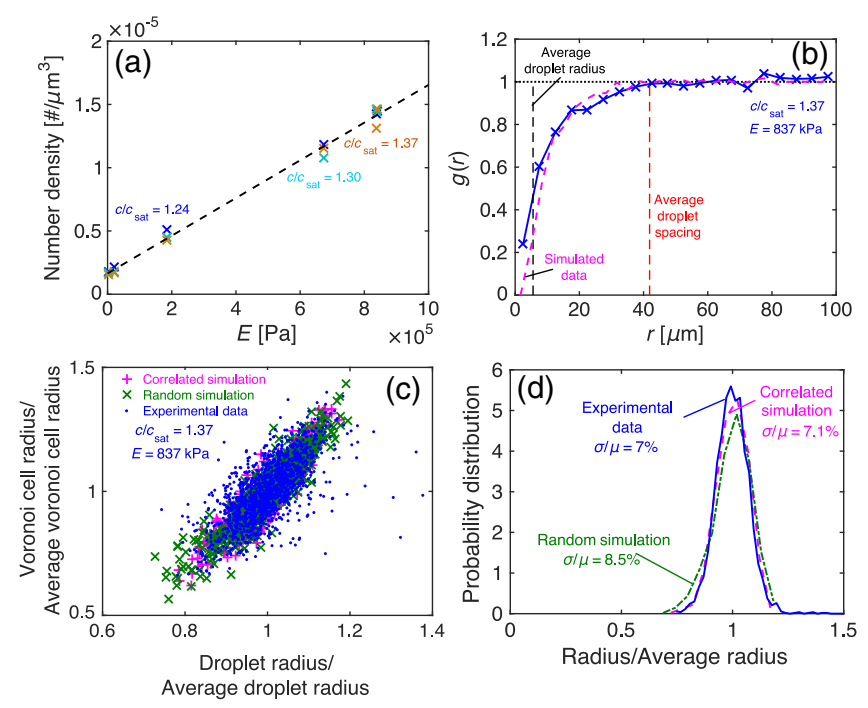

FIG. 5. Correlated nucleation and growth. (a) The number density of droplets depends on stiffness but not saturation. The dashed line is the line of best fit to the data. (b) The paircorrelation function $g(r)$ of droplet centers for a typical experiment (blue crosses) shows that nuclei do not form near each other. The range of internuclei repulsions is comparable to the interdroplet distance. The pink line shows the $g(r)$ for nuclei positions in our Monte Carlo growth simulation. Note that the experimental measurements of $g(r)$ are only accurate for $r \gtrsim 5 \mu \mathrm{m}$ because of the inaccuracy of out-of-plane tracking of droplet centers. (c) Droplet sizes are strongly correlated with the radius of their Voronoi cell. Blue: Data from the experiment in (b). Green and pink points show results of growth simulations with random and correlated nuclei positions. (d) Droplet size distributions for the experimental data in (b) (blue) and simulations with random (green) or correlated (pink) positions.

Voronoi construction. The Voronoi cell associated with a nucleus is the collection of points that are closer to it than any other nucleus. In general, this is a complex polyhedral shape. We reduce it to a single length scale by taking the cube root of the cell volume, or Voronoi radius, $r_{v}$. As shown in Fig. 5(c), the Voronoi radius is strongly correlated to the droplet size. Thus, droplets in tight clusters tend to be smaller than more widely spaced droplets. This effect, if present in conventional liquid-liquid phase separation, is masked by Brownian motion and coalescence of droplets.

Monte Carlo simulations suggest that the correlation of droplet size and nuclei spacing is a natural consequence of the diffusion-limited growth of droplets. Using Brownian dynamics, we simulated the diffusion and capture of solute molecules by a number of fixed nuclei. In one case, the nuclei were positioned completely randomly; in the other case, the nuclei positions were selected to have the same $g(r)$ as experiments. In both cases, we find a pronounced correlation between $r_{v}$ and droplet radii, as shown by the green (random) and pink (correlated) points in Fig. 5(c), which nicely match the experimentally observed correlation (blue). However, the simulation with correlated 
nucleation positions shows a narrower spread in $r_{v}$ and droplet radii, which closely matches the experiments, as shown in Fig. 5(d). Thus, long-range interactions of nuclei make the structure around each nucleus more regular and result in more uniform droplet radii.

Together, these results suggest that understanding the nucleation process is crucial to gaining a quantitative understanding of droplet size and monodispersity.

\section{ISOTHERMAL PHASE SEPARATION IN HYDROGELS}

To show the generality of uniform droplet production by phase separation in a polymer network, we demonstrate its efficacy for a distinct form of phase separation and diverse polymeric networks.

Previously, we supersaturated the system by quenching a binary liquid mixture with a change of temperature. Now, we drive the phase separation of a solute by changing the composition of a solvent mixture [7,43]. This form of phase separation is familiar from aperitifs like ouzo and pastis. In these drinks, fragrant anise oil is solubilized in water by a high concentration of ethanol [44]. When water is added to the drink, the mixture becomes unstable, and oil-rich droplets nucleate and grow.

We use three hydrogels with distinct chemistries: a chemically cross-linked gel (polydimethylacrylamide or PDMA), a thermally set physical gel (gellan gum), and an ionically cross-linked physical gel (alginate). In each case, we soak the hydrogel in a large volume of a stable mixture of water/ethanol/anise oil to exchange the solvent [see Fig. 1(b)]. After this, we cover the gel with deionized water, which diffuses inward and initiates phase separation. The resulting oil droplets are shown in Fig. 6. As with the fluorinated-oil/silicone system, we see rather uniform droplet distributions in the different materials (see Ref. [37] for distributions), especially in the PDMA and gellan gum samples, which we expect to be much more uniform gels than alginate [45].

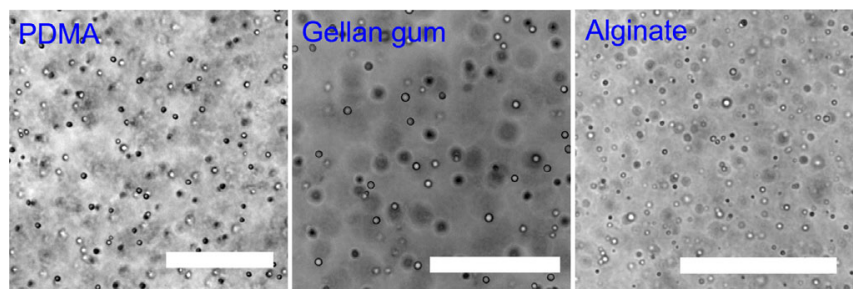

FIG. 6. Arrested isothermal phase separation in diverse hydrogels. Here, anise oil droplets nucleated and grew in an ethanolwater mixture (i.e., the Ouzo effect). Micrographs of dispersed droplets in (a) PDMA, a covalently cross-linked gel; (b) gellan gum, a thermally set, physically cross-linked gel; and (c) alginate, an ionically cross-linked physical gel. All droplets are stable against coalescence. Droplets in PDMA and gellan are reasonably monodisperse (see Ref. [37]). The scale bars are all $40 \mu \mathrm{m}$ wide.

\section{OPEN QUESTIONS}

While we have outlined the essential phenomenology and highlighted key thermodynamic and mechanical aspects of the process, much work must be done to yield a quantitative understanding. The central question is how an increase in the cross-linking density leads to an increase of nucleation sites. There are two key aspects to nucleation-its spatial distribution and its rate. The spatial distribution is likely to be set by the fact that when a droplet nucleates and starts to grow, it will deplete the surrounding material of solute, making it less likely for a nearby nucleus to appear. The control of nucleation rate is more complex, as it will depend on a range of factors, such as cooling rate and the ability of droplets to nucleate within the (nanometric-scale) mesh of the polymer gel. One simple possibility, suggested by the monotonic increase of nucleation sites with cross-linking density, is that the crosslinking sites themselves serve as heterogeneous nucleation sites. However, this appears to be at odds with our expectation that droplets do not wet the polymer network.

The mechanical aspects of this process demand further exploration. We have shown that the mechanical properties of the gel alter the phase diagram. However, there are a number of interesting further questions and possibilities. While elastic cavitation theory predicts a balance of elastic forces and condensation pressure only for droplets that are modestly larger than the mesh size, it is clear that strain-stiffening networks can suppress droplet growth and limit droplet size over a wider range of driving pressures. Furthermore, elastic forces could create an elastic Ostwald ripening effect. In classic Ostwald ripening, surface tension favors the collapse of small drops to feed big ones. Here, we anticipate that elastic forces in strain-stiffening networks could drive transport of the condensed phase from big drops to small ones. The balance of these two effects could lead to a mechanically defined equilibrium droplet size. However, precise quantitative theories of these effects will be challenging since the deformations are so large-here, the final droplets are about 3 orders of magnitude larger than the network mesh size. Therefore, there must be strong nonlinear or irreversible deformations - especially in the vicinity of the droplet - which are bound to be strongly rate dependent [32,46-48]. Finally, in all of our experiments, droplets appear to exclude the chains of the polymer network as they grow. However, we can envisage situations where phase-separating droplets could (partially) wet the network so that this would not necessarily occur. It is interesting to wonder how this would modify the process and what precise conditions are needed for the polymer network to always be excluded.

\section{CONCLUSIONS}

We have shown that phase separation of a solvent mixture in a cross-linked polymer network is a simple 
bulk technique to create uniform droplets. The size of the resulting droplets depends on a range of factors, including the cross-linking density of the polymer network, the supersaturation, and the quench rate. This process for the production of monodisperse droplets in bulk has broad potential applications. For example, it could be used to create quantities of composites with well-defined microstructures that enable novel optical or mechanical properties $[16,36]$, without the expense of using monodisperse colloidal templates (e.g., Ref. [40]). Additionally, by using a monomer as the phase-separating component, it can offer a flexible route for the synthesis of uniform microparticles. This process separates the control of particle size from polymerization kinetics and thus may allow a greater level of control over final particle size than other techniques.

Our results suggest a potential role for polymer networks in the regulation of membraneless organelles within living cells. Consider phase-separating macromolecules with molecular weights in the range of 75-600 kDa. Equation (1) suggests they can exert pressures of order 4-33 kPa. These pressures are comparable to the stiffness of many cytoskeletal networks, which suggests that living cells may be able to regulate the nucleation and growth of membraneless organelles through their mechanical properties.

\section{MATERIALS AND METHODS}

\section{A. Silicone-gel preparation and characterization}

We prepare silicone gels following the recipe of Ref. [36], except where stated otherwise. These are prepared with a mixture of vinyl-terminated polydimethylsiloxane chains (DMS-V31, Gelest), cross-linker: trimethylsiloxane terminated (25\%-35\% methylhydrosiloxane)-dimethylsiloxane copolymer (HMS-301, Gelest Inc.), and catalyst (SIP6831.2, Gelest). Stiffness is tuned by varying the proportion of cross-linker in the gel between $0.8 \%$ and $3.3 \%$. We measure Young's modulus of each sample by making an additional bulk sample and performing a compression test. For this test, the silicone gel is formed in a cylindrical plastic mold (depth $10 \mathrm{~mm}$, radius $15 \mathrm{~mm}$ ) and then indented with a 1-mm radius, cylindrical indenter using a TA.XT plus texture analyzer with a 500-g load cell (Stable Micro Systems).

For some of the light-scattering experiments, we used a different variety of silicone gel (Sylgard 184, Dow Corning), as this is less sticky and thus easier to stretch manually. For these samples, stiffness was again tuned by varying the ratio of base to cross-linker.

Silicone-gel samples are made by coating the inside of glass-bottomed petri dishes with a thin, $O(1-\mathrm{mm})$-thick film of silicone gel. Silicone-gel samples are then saturated with fluorinated oil (Fluorinert FC770, Fluorochem) at an elevated temperature $T_{e}$.
The solubility of fluorinated oil in the gels was measured by weighing samples of silicone gels of two different stiffnesses $(E=20,840 \mathrm{kPa})$ before and after saturation at $23^{\circ} \mathrm{C}, 40^{\circ} \mathrm{C}$, and $55^{\circ} \mathrm{C}$. No significant change in solubility was found with $E$. See Ref. [37] for further details.

\section{B. Hydrogel preparation}

PDMA samples were fabricated by free-radical polymerization of N-N-dimethylacrylamide (99\% DMA, SigmaAldrich) with N,N'-methylenebisacrylamide (99\% MBA, Sigma-Aldrich) as a cross-linker. Ammonium persulphate ( $\geq 98 \%$ APS, Fisher-Bio) and N,N,N',N'-tetramethylethylenediamine (99.8\% TEMED, Apollo Scientific) were used as the redox/initiator system (e.g., Ref. [49]). All chemicals were used as received, and the reaction was performed in a nitrogen glovebox to avoid inhibition of the reaction by oxygen. We prepared fresh stock solutions with deionized water $(18.2 \mathrm{M} \Omega . \mathrm{cm})$ as follows: $30 \% \mathrm{w} / \mathrm{v}$ DMA, $2 \% \mathrm{w} / \mathrm{v}$ MBA, $10 \% \mathrm{w} / \mathrm{v}$ APS, and 10\% w/v TEMED. To make $10 \mathrm{~mL}$ of PDMA, we combined 2.5-mL of DMA solution, $0.8-\mathrm{mL}$ of MBA solution, and 6.64-mL of deionized water before gently mixing. Then we added $0.03-\mathrm{mL}$ of TEMED solution and $0.03-\mathrm{mL}$ of APS solution, mixing the sample gently after each addition. Finally, the sample was poured into a mold and left to completely cure.

Gellan-gum samples were fabricated from $0.5 \mathrm{wt} \%$ gellan gum (BIPG434, Apollo Scientific Ltd.), 59.5 wt $\%$ deionized water, and $40 \mathrm{wt} \%$ ethanol $(99.8 \%$, Chemie Brunschwig). The gum powder is mixed with water, heated to $90^{\circ} \mathrm{C}$, and stirred until fully dissolved. Ethanol is then added dropwise while stirring, and the sample is removed from the heat. Typically, we see a separation of the sample into an upper, cloudy part and a lower, transparent part. We pipette the lower, clear phase into petri dishes and allow it to gel. Finally, the gel is soaked in a $50-500-\mathrm{mM} \mathrm{CaCl}_{2}$ solution (98\% Sigma-Aldrich) to allow full cross-linking by the calcium ions.

Alginate-gel samples were fabricated from 2 wt $\%$ sodium alginate (Acros) in deionized water. This mixture is gently placed in contact with a $100-\mathrm{mM} \mathrm{CaCl}_{2}$ solution and left for long enough to allow the calcium ions to diffuse into and cross-link the alginate solution (e.g., Ref. [50]).

\section{Controlled cooling apparatus}

To repeatedly cool our samples in a precise manner, we built a computer-controlled cooling chamber. This consists of a $7 \times 7 \times 7-\mathrm{cm}$, insulated, aluminium-foil-lined, insulated chamber on a 4-mm-thick aluminium plate, which is mounted on a $12 \mathrm{~V}$ peltier element. A fan in the chamber circulates the air inside to maintain a uniform temperature. A thermistor (NTC $30 \mathrm{k} \Omega$, Amphenol Advanced Sensors) is embedded in the aluminium plate. This is connected via a PID controller (programmed on an Arduino microcontroller) to the peltier element, allowing the chamber to be set at a defined temperature. The air temperature inside the 
chamber is then measured with an Arduino temperature sensor (TMP36).

To cool the sample, we preheat the chamber, insert the sample, and wait for the temperature to equilibrate. We then set the sample to cool at a fixed rate until it reaches room temperature, $23^{\circ} \mathrm{C}$.

\section{3D image analysis}

For each sample, we recorded a stack of images through a section of a sample with depth of approximately $250 \mu \mathrm{m}$ at $2.5-\mu \mathrm{m}$ intervals. We performed bright-field microscopy with a Nikon Eclipse Ti2 microscope and either a 20x (NA 0.45) or 40x (NA 0.6) air objective (see Ref. [37] for examples of stacks). The images are then analyzed using a bespoke programme with MATLAB's imfindcircles functionality to identify, for each droplet in the stack, its radius and location in 3D. There is some ambiguity as to what to define as the precise edge of a droplet in images, as droplet edges appear as a dark, $O(\mu \mathrm{m})$-thick ring. We choose to measure radii as being at the outer edge of this ring. This choice gives excellent reliability in relative measurements of droplet size. However, there may be a systematic error of up to about $1.5 \mu \mathrm{m}$. We calculated the volume of the Voronoi cell for each droplet using MATLAB's voronoi function. The Voronoi cell of a droplet is the collection of points in 3D that are closer to its center than the center of any other droplet.

\section{E. Growth simulations}

We explored the effect of internuclei structural correlations on the growth of droplets using Monte Carlo simulations. In the first step, we positioned $10^{3}$ nuclei in a cube with periodic boundary conditions, either by placing the nuclei independently or using the Metropolis algorithm to generate a pattern of points with the same $g(r)$ as observed in experiments. In the second step, we released $10^{6}$ molecules, which underwent a random walk until they were captured by a nucleus. By counting how many molecules were captured by each nucleus, we were able to determine the final size of each nucleus. For simplicity, the target radius for each nucleus was fixed throughout the simulation so that the volume fraction of the targets was $1.25 \%$.

\section{F. Optical experiments}

We observed the scattering of red, laser light from a HeNe laser (Thorlabs HNL020L) through $O(1-\mathrm{mm})$-thick samples. The scattering pattern was projected onto a sheet of thin, tracing paper, which was then imaged from behind with a Nikon 1 J5 camera.

We observed the scattering of white light by positioning a white-light LED (Apple, iPhone 6) behind a sample and then imaging the scattering pattern with the same camera.

\section{ACKNOWLEDGMENTS}

We acknowledge helpful discussions with Alain Goriely and Ian Griffiths and funding from the Swiss National Science Foundation (Grants No. 200021_172824 and No. 200021_172827). The authors declare no competing financial interests.

R. W. S., L. A. W., and E.R. D. designed experiments. R. W. S., T. S., N. F., M. I., K. S.-M., and Q. X. performed experiments. R. W. S., L. A. W., and E. R. D. analyzed data. R. W. S., L. A. W., and E.R.D. developed the theory. R. W. S. and E. R. D. wrote the paper.

[1] C.P. Brangwynne, Soft Active Aggregates: Mechanics, Dynamics and Self-Assembly of Liquid-like Intracellular Protein Bodies, Soft Matter 7, 3052 (2011).

[2] D. M. Mitrea and R. W. Kriwacki, Phase Separation in Biology; Functional Organization of a Higher Order, Cell Commun. Signaling 14, 1 (2016).

[3] J. Willard Gibbs, The Scientific Papers of J. Willard Gibbs, Vol. 1 (Longmans, Green, New York, 1906).

[4] V. J. Anderson and H. N. W. Lekkerkerker, Insights into Phase Transition Kinetics from Colloid Science, Nature (London) 416, 811 (2002).

[5] M. Avrami, Kinetics of Phase Change I. General Theory, J. Chem. Phys. 7, 1103 (1939).

[6] J. W. Cahn, Phase Separation by Spinodal Decomposition in Isotropic Systems, J. Chem. Phys. 42, 93 (1965).

[7] V. K. LaMer and R. H. Dinegar, Theory, Production and Mechanism of Formation of Monodispersed Hydrosols, J. Am. Chem. Soc. 72, 4847 (1950).

[8] A. A. Hyman, C. A. Weber, and F. Jülicher, Liquid-Liquid Phase Separation in Biology, Annu. Rev. Cell Dev. Biol. 30, 39 (2014).

[9] A. Molliex, J. Temirov, J. Lee, M. Coughlin, A. P. Kanagaraj, H. J. Kim, T. Mittag, and J. P. Taylor, Phase Separation by Low Complexity Domains Promotes Stress Granule Assembly and Drives Pathological Fibrillization, Cell 163, 123 (2015).

[10] A. Patel, H. O. Lee, L. Jawerth, S. Maharana, M. Jahnel, M. Y. Hein, S. Stoynov, J. Mahamid, S. Saha, T. M. Franzmann et al., A Liquid-to-Solid Phase Transition of the ALS Protein FUS Accelerated by Disease Mutation, Cell 162, 1066 (2015).

[11] J. A. Riback, C. D. Katanski, J. L. Kear-Scott, E. V. Pilipenko, A. E. Rojek, T. R. Sosnick, and D. A. Drummond, StressTriggered Phase Separation Is an Adaptive, Evolutionarily Tuned Response, Cell 168, 1028 (2017).

[12] L. Zhu and C. P. Brangwynne, Nuclear Bodies: The Emerging Biophysics of Nucleoplasmic Phases, Curr. Opin. Cell Biol. 34, 23 (2015).

[13] D. Zwicker, M. Decker, S. Jaensch, A. A. Hyman, and F. Jülicher, Centrosomes Are Autocatalytic Droplets of Pericentriolar Material Organized by Centrioles, Proc. Natl. Acad. Sci. U.S.A. 111, E2636 (2014).

[14] C. P. Brangwynne, P. Tompa, and R. V. Pappu, Polymer Physics of Intracellular Phase Transitions, Nat. Phys. 11, 899 (2015). 
[15] C. P. Brangwynne, T. J. Mitchison, and A. A. Hyman, Active Liquid-like Behavior of Nucleoli Determines Their Size and Shape in Xenopus Laevis Oocytes, Proc. Natl. Acad. Sci. U.S.A. 108, 4334 (2011).

[16] E. R. Dufresne, H. Noh, V. Saranathan, S. G. J. Mochrie, H. Cao, and R. O. Prum, Self-Assembly of Amorphous Biophotonic Nanostructures by Phase Separation, Soft Matter 5, 1792 (2009).

[17] M. L. Gardel, J. H. Shin, F. C. MacKintosh, L. Mahadevan, P. Matsudaira, and D. A. Weitz, Elastic Behavior of Cross-linked and Bundled Actin Networks, Science 304, 1301 (2004).

[18] Q. Wen and P. A. Janmey, Polymer Physics of the Cytoskeleton, Curr. Opin. Solid State Mater. Sci. 15, 177 (2011).

[19] J. H. Holtz and S. A. Asher, Polymerized Colloidal Crystal Hydrogel Films as Intelligent Chemical Sensing Materials, Nature (London) 389, 829 (1997).

[20] M. Kolle, A. Lethbridge, M. Kreysing, J. J. Baumberg, J. Aizenberg, and P. Vukusic, Bio-inspired Band-gap Tunable Elastic Optical Multilayer Fibers, Adv. Mater. 25, 2239 (2013).

[21] M. Feric and C. P. Brangwynne, A Nuclear F-actin Scaffold Stabilizes RNP Droplets Against Gravity in Large Cells, Nat. Cell Biol. 15, 1253 (2013).

[22] E. M. Herzig, K. A. White, A. B. Schofield, W. C. K. Poon, and P. S. Clegg, Bicontinuous Emulsions Stabilized Solely by Colloidal Particles, Nat. Mater. 6, 966 (2007).

[23] H. Tanaka, Viscoelastic Phase Separation, J. Phys. Condens. Matter 12, R207 (2000).

[24] P. Atkins and J. De Paula, Elements of Physical Chemistry (Oxford University, New York, 2013).

[25] Q. Liu and Z. Suo, Osmocapillary Phase Separation, Extreme Mech. Lett. 7, 27 (2016).

[26] S. Cai, K. Bertoldi, H. Wang, and Z. Suo, Osmotic Collapse of a Void in an Elastomer: Breathing, Buckling and Creasing, Soft Matter 6, 5770 (2010).

[27] T. D. Wheeler and A.D. Stroock, The Transpiration of Water at Negative Pressures in a Synthetic Tree, Nature (London) 455, 208 (2008).

[28] M. J. Schnitzer, K. Visscher, and S. M. Block, Force Production by Single Kinesin Motors, Nat. Cell Biol. 2, 718 (2000).

[29] J. M. Ball, Discontinuous Equilibrium Solutions and Cavitation in Nonlinear Elasticity, Phil. Trans. R. Soc. A 306, 557 (1982).

[30] A. N. Gent, Rubber and Rubber Elasticity: A Review, J. Polym. Sci., Polym. Symp. 48, 1 (1974).

[31] A. N. Gent and P. B. Lindley, Internal Rupture of Bonded Rubber Cylinders in Tension, Proc. R. Soc. A 249, 195 (1959).

[32] A. N. Gent and C. Wang, Fracture Mechanics and Cavitation in Rubber-like Solids, J. Mater. Sci. 26, 3392 (1991).
[33] S. Kundu and A. J. Crosby, Cavitation and Fracture Behavior of Polyacrylamide Hydrogels, Soft Matter 5, 3963 (2009).

[34] J. A. Zimberlin, N. Sanabria-DeLong, G. N. Tew, and A. J. Crosby, Cavitation Rheology for Soft Materials, Soft Matter 3, 763 (2007).

[35] J. Zhu, T. Li, S. Cai, and Z. Suo, Snap-through Expansion of a Gas Bubble in an Elastomer, J. Adhes. 87, 466 (2011).

[36] R. W. Style, R. Boltyanskiy, B. Allen, K. E. Jensen, H. P. Foote, J. S. Wettlaufer, and E. R. Dufresne, Stiffening Solids with Liquid Inclusions, Nat. Phys. 11, 82 (2015).

[37] See Supplemental Material at http://link.aps.org/ supplemental/10.1103/PhysRevX.8.011028 for further experimental details and supplemental videos.

[38] C. F. Bohren and D. R. Huffman, Absorption and Scattering of Light by Small Particles (John Wiley \& Sons, New York, 2008).

[39] C. Mätzler, Technical Report No. 2002-11, Institut für Angewandte Physik, 2002.

[40] H. Fudouzi and T. Sawada, Photonic Rubber Sheets with Tunable Color by Elastic Deformation, Langmuir 22, 1365 (2006).

[41] E. Lepeltier, C. Bourgaux, and P. Couvreur, Nanoprecipitation and the "Ouzo Effect": Application to Drug Delivery Devices, Adv. Drug Delivery Rev. 71, 86 (2014).

[42] H. T. Meryman, Mechanics of Freezing in Living Cells and Tissues, Science 124, 515 (1956).

[43] S. A. Vitale and J. L. Katz, Liquid Droplet Dispersions Formed by Homogeneous Liquid-Liquid Nucleation: "The Ouzo Effect," Langmuir 19, 4105 (2003).

[44] H. Tan, C. Diddens, P. Lv, J. G. M. Kuerten, X. Zhang, and D. Lohse, Evaporation-Triggered Microdroplet Nucleation and the Four Life Phases of an Evaporating Ouzo Drop, Proc. Natl. Acad. Sci. U.S.A. 113, 8642 (2016).

[45] M. Heinemann, H. Meinberg, J. Büchs, H.-J. Koß, and M. B. Ansorge-Schumacher, Method for Quantitative Determination of Spatial Polymer Distribution in Alginate Beads Using Raman Spectroscopy, Appl. Spectrosc. 59, 280 (2005).

[46] C. Creton and M. Ciccotti, Fracture and Adhesion of Soft Materials: AReview, Rep. Prog. Phys. 79, 046601 (2016).

[47] S. B. Hutchens, S. Fakhouri, and A. J. Crosby, Elastic Cavitation and Fracture via Injection, Soft Matter 12, 2557 (2016).

[48] C. W. MacMinn, E. R. Dufresne, and J. S. Wettlaufer, FluidDriven Deformation of a Soft Granular Material, Phys. Rev. X 5, 011020 (2015).

[49] N. Orakdogen and O. Okay, Influence of the Initiator System on the Spatial Inhomogeneity in Acrylamide-based Hydrogels, J. Appl. Polym. Sci. 103, 3228 (2007).

[50] B. Joddar, E. Garcia, A. Casas, and C. M. Stewart, Development of Functionalized Multi-walled CarbonNanotube-Based Alginate Hydrogels for Enabling Biomimetic Technologies, Sci. Rep. 6, 32456 (2016). 